\title{
A NONLINEAR INDICIAL PREDICTION TOOL FOR UNSTEADY AERODYNAMIC MODELING
}

\author{
Patrick H. Reisenthel ${ }^{*}$ and Matthew T. Bettencourt ${ }^{*}$ \\ Nielsen Engineering \& Research, Inc. \\ Mountain View, CA \\ James H. Myatt ${ }^{\S}$ and Deborah S. Grismer ${ }^{\mathbb{I I}}$ \\ Air Force Research Laboratory / Aeronautical Sciences Division \\ Wright-Patterson AFB, OH
}

\begin{abstract}
The present paper describes a new tool kit which can be used to model the time-dependent response of nonlinear systems. The Indicial Prediction System (IPS) applies nonlinear indicial theory to solve complex unsteady problems, such as those associated with nonlinear aerodynamic phenomena during maneuver of aircraft. The functionality of this system and its capabilities are described through numerous examples. An important demonstration of the method is its application to the prediction of the aerodynamic loads on a 65-degree delta wing undergoing forced body-axis rolling motions at high angles of attack.
\end{abstract}

\section{NOMENCLATURE}

\section{Symbols and abbreviations}

b Wing span

c Wing chord

$\mathrm{C}_{1} \quad$ Body-axis rolling moment coefficient, nondimensionalized with respect to $q S b$

CS Critical State

CSR Critical-state Response

DEP Dependent variable

DOF Degree of Freedom

$f \quad$ Aerodynamic load (generic)

$\mathrm{f} \quad$ Frequency

$\mathrm{f}_{\downarrow} \quad$ Indicial response of $f$ with respect to $\phi$

$\stackrel{\phi}{f} \quad$ Indicial response of $f$

$\widetilde{h} \quad$ Deficiency function $(\widetilde{h} \equiv \widetilde{f}(t)-\widetilde{f}(t \rightarrow \infty))$

$\begin{array}{ll}H & \text { Heaviside step function } \\ \mathrm{IE} & \text { Indicial Extraction } \\ \mathrm{IP} & \text { Indicial Prediction } \\ \mathrm{IPS} & \text { Indicial Prediction System } \\ \mathrm{IR} & \text { Indicial Response } \\ k & \text { Reduced frequency }\left(k \equiv \omega \mathrm{b} / 2 \mathrm{U}_{\infty}\right) \\ n_{\text {harm }} & \text { Number of retained harmonics } \\ \mathrm{NIR} & \text { Nonlinear indicial response } \\ N_{\phi} & \text { Number of nodal extraction roll angles } \\ \mathrm{p} & \text { Roll rate } \\ \mathrm{q} & \text { Dynamic pressure } \\ \mathrm{QS} & \text { Quasistatic } \\ \mathrm{rms} & \text { Root mean square } \\ \mathrm{S} & \text { Wing area } \\ s g n & \text { Sign } \\ \mathrm{t} & \text { Time } \\ \mathrm{T} & \text { Period of oscillation } \\ \mathrm{U}_{\infty} & \text { Freestream velocity } \\ \alpha & \text { Angle of attack } \\ \delta & \text { Dirac delta function } \\ \delta \mathrm{C}_{\mathrm{l}} / \delta \phi & \text { Indicial response of rolling moment with } \\ \Delta \mathrm{f} & \text { respect to roll angle } \\ \Delta \mathrm{f}^{\mathrm{CS}} & \text { Build-up of generic aerodynamic load, } f \\ \epsilon & \text { Critical-state response of } f \\ \phi & \text { Boundary condition (generic) } \\ \sigma & \text { Roll angle } \\ \tau & \text { Support sting angle } \\ \tau & \text { Time constant; alternatively, auxiliary time } \\ \tau \mathrm{c} & \text { integration variable in integrals } \\ \omega & \text { Time at which critical state is crossed } \\ & \text { Angular frequency } \\ & \end{array}$

$\mp$ Chief Scientist, Member AIAA

* Research Scientist

$\S$ Aerospace Engineer, Member AIAA

- Aerospace Engineer, Member AIAA

Copyright $\odot 1998$ by Nielsen Engineering \& Research, Inc.

Published by the American Institute of Aeronautics, Inc. with permission. 
$\xi \quad$ Parameter denoting dependence on prior motion history

$\xi_{j}(\mathrm{t}) \quad$ Basis function

$\begin{array}{ll}\text { Subscripts } \\ \text { c } & \text { Critical } \\ \text { CS } & \text { Critical State } \\ \text { DR } & \text { Deficiency Response } \\ \text { dyn } & \text { Dynamic } \\ \text { QS } & \text { Quasistatic } \\ \infty & \text { Time-asymptotic value (except for } \mathrm{U}_{\infty} \text { ) }\end{array}$

\section{Superscripts}

CS Critical State

dyn Dynamic component

v Vortical

- Derivative with respect to time

- $\quad$ Second derivative with respect to time

$\sim \quad$ Indicial or deficiency function

\section{INTRODUCTION}

In recent years, it has been possible to integrate the flight-dynamics equations fairly efficiently using linearized aerodynamics which are occasionally supplemented with ad hoc methods (i.e., semiempirical simulations or wind tunnel data) to include nonlinear unsteady aerodynamic effects. However, with the expanded flight envelopes being considered for future maneuvering aircraft, it has become increasingly important to be able to model and predict nonlinear, unsteady aerodynamics. This includes the prediction of the aerodynamic response in the presence of flow separation, shock movement, and vortex bursting at high angles of attack and/or high angular rates.

Future fighter aircraft will be required to perform controlled maneuvers well beyond traditional aircraft limits, for example, pitch up and flight at high angles of attack, rapid point-to-shoot, and other close-in combat maneuvers. These advanced maneuvers demand the use of aerodynamic methods capable of predicting characteristics of the nonlinear post-stall regime for multiaxis motions at extremely high rates. At present, the only methods of this scope are NavierStokes methods. However, their use in flight simulations remains impractical at this time.

One method which has the potential to circumvent some of the present difficulties is the application of nonlinear indicial theory [1,2]. An example of the latter (the Indicial Prediction System described herein) combines features of flexibility in modeling, good execution speed, and high fidelity representation.

\section{OBJECTIVE AND APPROACH}

The objective of this paper is to provide an overall description of the Indicial Prediction System, with an emphasis on its use as a tool box for the aerodynamicist and control system designer. This paper is organized as follows. First, a brief theoretical background is given. This is followed by an overview of the system, including the basic algorithms and examples illustrating some of the current capabilities. Code validation results are then presented. Finally, the complete system is applied to a subset of the U.S. Air Force Research Laboratory (formerly USAF/WL) and Canadian Institute for Aerospace Research (IAR) 65-degree delta wing database.

\section{INDICIAL THEORY}

The indicial approach is based on the concept that a characteristic flow variable $f(t)$, which describes the state of the flow, can be linearized with respect to its boundary condition (or forcing function), $\epsilon(t)$, if the variation of $f(t)$ is a smooth function of $\epsilon(\mathrm{t})$. This allows the representation of $f(t)$ in a Taylor series about some value $\epsilon=\epsilon_{0}$; thus

$$
f(t)=f(0)+\left.\Delta \varepsilon \frac{\partial f}{\partial \varepsilon}\right|_{\varepsilon=\varepsilon_{0}}+\cdots
$$

If the response $\partial f / \partial \boldsymbol{\varepsilon}$ depends only on the elapsed time from the perturbation $\Delta \epsilon$ (a linear time-invariant response) then it may be shown [3] that the formal solution for $f(t)$ is

$$
f(t)=\tilde{f}(t) \varepsilon(0)+\int_{0}^{t} \frac{d \boldsymbol{\varepsilon}}{d \tau} \tilde{f}(t-\tau) d \tau
$$

where $\tilde{f}(t)=\left.\frac{\partial f}{\partial \varepsilon}\right|_{\varepsilon=\varepsilon_{0}}$.

Hence, if the forcing function (i.e., the boundary condition $\epsilon$ ) is known and if $\widetilde{f}$ (the indicial response) is known from some computation or experiment, then Eq. (1) gives the value of $f(t)$ for any schedule of the boundary condition $\epsilon(\mathrm{t})$ without the need to compute $f$ from first principles. 
The basic idea behind the use of nonlinear indicial response theory [1,2] is that the linear formalism, Eq. (1), can be retained in the form of a generalized superposition integral, provided that the nonlinear indicial response $f$ is now taken to be a functional $\widetilde{f}(\epsilon(\xi) ; \mathrm{t}, \tau)$, where $\epsilon(\xi)$ denotes the dependence on the entire prior motion history. Furthermore, the nonlinear indicial theoretical formulation allows for the presence of aerodynamic bifurcations by splitting the integral, for example:

$$
\begin{array}{r}
f(t)=f(\varepsilon(\xi) ; t, 0)+\int_{0}^{\tau_{C}} \frac{d \varepsilon}{d \tau} \tilde{f}(\varepsilon(\xi) ; t, \tau) d \tau \\
+\Delta f^{C S}\left(t ; \varepsilon\left(\tau_{C}\right)\right)+\int^{t} \frac{d \varepsilon}{d \tau} \tilde{f}(\varepsilon(\xi) ; t, \tau) d \tau
\end{array}
$$

where the nonlinear indicial function $\widetilde{f}(\epsilon(\xi) ; t, \tau)$ is defined as the following Fréchet derivative:

$$
\begin{aligned}
& \tilde{f}(\varepsilon(\xi) ; t, \tau)=\lim _{\Delta \varepsilon \rightarrow 0} \frac{\Delta f(t)}{\Delta \varepsilon} \\
& =\lim _{\Delta \varepsilon \rightarrow 0}\left[\frac{f(\varepsilon(\xi)+H(\xi-\tau) \Delta \varepsilon)-f(\varepsilon(\xi))}{\Delta \varepsilon}\right]
\end{aligned}
$$

and $\Delta \mathrm{f}^{\mathrm{CS}}\left(\mathrm{t} ; \epsilon\left(\tau_{\mathrm{c}}\right)\right)$ is the so-called jump response associated with crossing the bifurcation at time $\tau_{\mathrm{c}}$.

A critical state is defined [4] as a transition from one equilibrium flow state to another and is often associated with a discontinuity in the static aerodynamic loads and/or their derivatives [5]. The associated transient response, $\Delta \mathrm{f}^{\mathrm{CS}}\left(\mathrm{t} ; \epsilon\left(\tau_{\mathrm{c}}\right)\right)$, is referred to either as the critical-state response (CSR) or the jump response.

\section{INDICIAL PREDICTION SYSTEM}

The Indicial Prediction System is essentially the union of two codes: an indicial prediction (IP) code and an indicial extraction (IE) code. The indicial prediction code uses a database of indicial and critical-state responses to predict the output of the system to more or less arbitrary inputs, while the indicial extraction code is responsible for the creation of this database from empirical data. Both modules are based on the concept of a nodal, parameterized representation of the nonlinear indicial response (References 6 and 7).

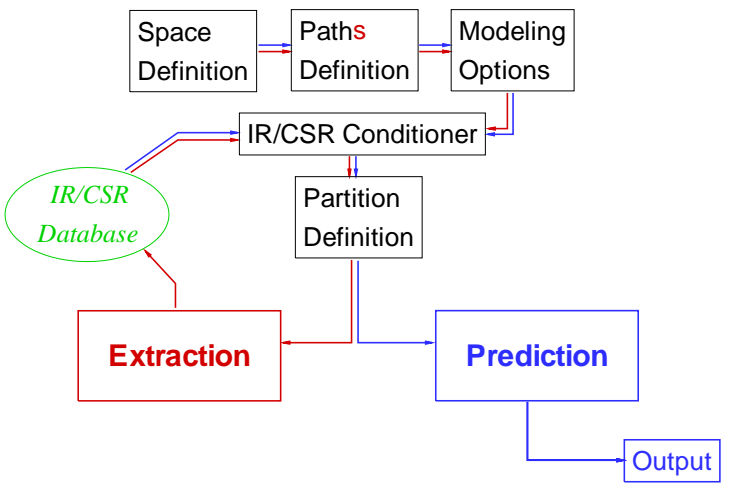

Fig. 1. The IE and IP Programs Share Common Module Components.

The overall structure of the IPS is given in Figure 1. This figure depicts the main components of the system, many of which are shared between the IE and IP modules. We will now briefly describe the functionality of each of the components.

The purpose of the space definition module is to define all possible dependent and independent variables for a given configuration or subcase of a given configuration. A configuration file is parsed for these variables, along with static (descriptive) parameters such as flow conditions and geometry. The configuration file also contains IR/CSR database information as well as textual information such as title and comment fields.

The path/maneuver definition module has two primary purposes: specification of inputs and, in the extraction case, specification of the "training" data used to identify the IR/CSR database. The inputs specification serves to optionally deactivate some of the a priori independent variables and to specify bounds on the retained variables. For instance, in a six degree-of-freedom simulation, one may specify that pitch-plane-only maneuvers are being considered. This identifies a subset (the active degrees of freedom) of the independent variables as timedependent. The motion source specification allows the program to identify the source of the motion as being a file (tabular form) or a shared object subroutine.

The modeling options module allows the user to experiment with various parameterizations of the indicial and critical-state responses. In some cases, this may even include the possibility that the desired parameterizations are not strictly consistent with the 
nominal parameterization expressed in the IR/CSR database (see below). "Modeling" in this context is defined as a series of decisions made by the user which include the choice of the dependent variables to be predicted $\left(\mathrm{DEP}_{\mathrm{i}}\right)$, the choice of which active degrees of freedom $\left(\mathrm{DOF}_{\mathrm{j}}\right)$ affect each of these dependent variables individually, and, for each of the retained $\left(\mathrm{DEP}_{\mathrm{i}}, \mathrm{DOF}_{\mathrm{j}}\right)$ combinations, how to calculate the contributions due to the indicial $\left(\operatorname{IR}_{\mathrm{ij}}\right)$ and criticalstate responses $\left(\mathrm{CSR}_{\mathrm{ij}}\right)$. In particular, each $\mathrm{IR}_{\mathrm{ij}}$ or $\mathrm{CSR}_{\mathrm{ij}}$ may be treated/overridden as quasistatic rather than dynamic, and each $\mathrm{IR}_{\mathrm{ij}} / \mathrm{CSR}_{\mathrm{ij}}$ may be parameterized in different ways (see Figure 2).

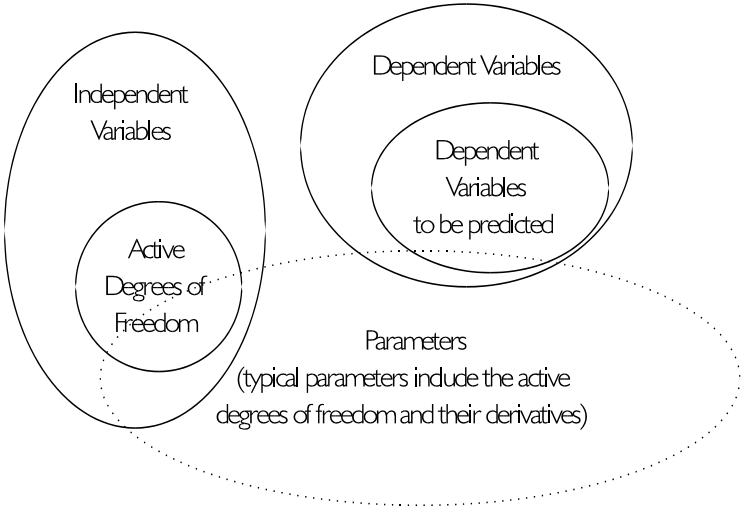

Fig. 2. Schematic Depicting the Relationship Between Parameters, Active Degrees of Freedom, Active Dependent Variables and the a priori Dependent and Independent Variables of the System.

The IR/CSR database consists of a number of files containing information relative to the known indicial and critical-state responses. Each IR or CSR file represents one or more nodal responses. Each file contains a header, followed by a data description. The data description consists of a list of dependent variables, one independent variable, and a list of parameters pertaining to this particular node. For example, the file might describe the nonlinear indicial response of $\mathrm{C}_{1}$ and $\mathrm{C}_{\mathrm{m}}$ (two dependent variables) with respect to $\phi$ (the independent variable), at $\phi=15^{\circ}$ (first parameter), $\mathrm{d} \phi / \mathrm{dt}=-0.01$ (second parameter), $\sigma=30^{\circ}$ (third parameter) and $\operatorname{sgn}\left(\mathrm{d}^{2} \phi / \mathrm{dt}^{2}\right)>0$ (fourth parameter). The responses per se are specified in two parts. The first item is the time-asymptotic or quasistatic value of the response (i.e., the aerodynamic derivative for each of the dependent variables). The second item is a description of the deficiency responses for each dependent variable (the deficiency response is the indicial response minus its time-asymptotic value). A deficiency response can be described either in tabular form or subroutine form. If its form is not specified, then the deficiency response is taken to be zero, meaning that the response is quasistatic.

The purpose of the IR/CSR conditioner is twofold. Its first function is to filter the contents of the IR/CSR database in order to establish a short list of IR/CSR nodes "likely" to participate in the interpolation process. Its second function, in the IP mode, is to resolve parameterization conflicts, thus maintaining the user's ability to execute the program even in cases where the contents of the IR/CSR database are not strictly consistent with the desired parameterizations being experimented with. This also allows the handling of heterogeneous databases. For example, it is conceivable that the IR/CSR database may have been created initially with certain parameterizations in mind, but then modified through the addition of extra responses with different (either fewer or more) parameterizations. In this case, the IR/CSR conditioner permits the program to function without having to regenerate the entire database.

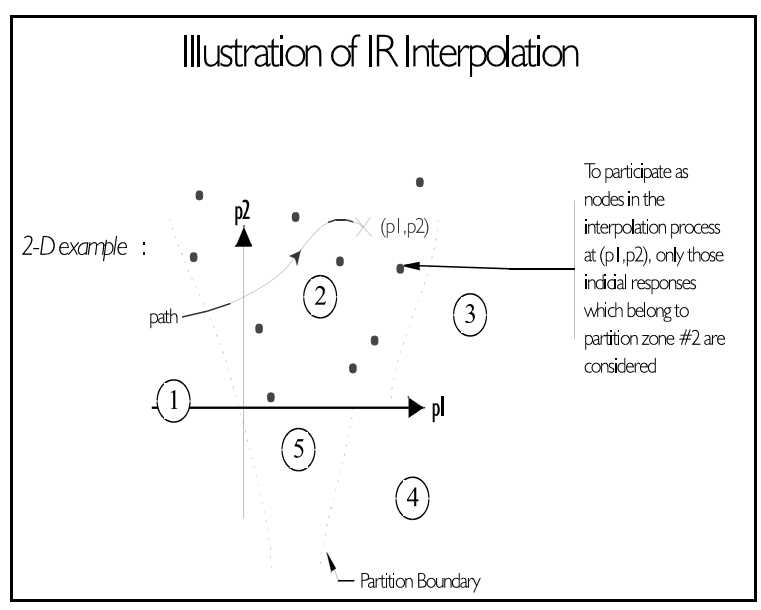

Fig. 3. Two-Dimensional Illustration of IR/CSR Space Partition.

At the end of the IR/CSR conditioner tasks, the useful portion of the database has been ingested and homogenized. One task remains to complete the modeling decisions: the definition of partitions of the IR space and the assignment of critical states. The tasks carried out in the modeling options module define the representation of the IR/CSR parameter space and its dimensionality. The Partition Definition Module creates partitions of the parameter space. The purpose of partitioning is to carve out 
sections of the parameter space within which likenodes are considered for functional interpolation (see Figure 3). Partition transitions are then classified according to whether or not they are to be modeled as critical states (Figure 4).

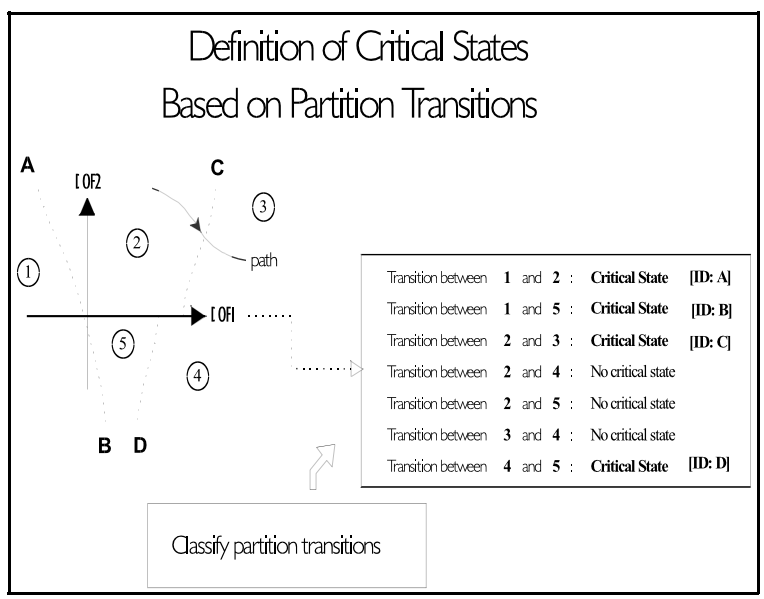

Fig. 4. Classification of Parameter Space Partitions as Critical States.

The Prediction Module and the Extraction Module are the core computational modules of the Indicial Prediction System. The task of extracting nonlinear indicial and critical-state responses from experimental data is a challenging one, and a separate paper (Reference 8) will be devoted to this topic. The prediction module is responsible for carrying out the nonlinear indicial theoretical prediction. Thus, it is responsible for integrating (in a generalized sense) the indicial and critical state contributions. The basic algorithm in the prediction module is as follows. For each dependent variable $\mathrm{DEP}_{\mathrm{i}}$, the predicted response is the sum of the effects of each participating active degree of freedom. Each of these effects are separated into so-called regular contributions (due to the indicial responses), and critical state contributions. Each contribution is further subdivided into quasistatic and dynamic components. The integration is done in two steps. The first step is the integration of the regular contributions. The update of each dependent variable is symbolically denoted

$D E P \leftarrow \sum_{D O F} \int_{0}^{t} I R_{Q S} D \dot{O O F} d \tau+\sum_{D O F} \int_{0}^{t} D R(t-\tau) \dot{D O F}(\tau) d \tau$ where $D R$ stands for the deficiency response $\left(D R \equiv I R-I R_{Q S}\right)$. The second step is to sum up all relevant critical-state response contributions. Again, the update of each computed dependent variable is symbolically denoted

$$
D E P \leftarrow D E P+\sum_{D O F}\left(\sum_{C S} C S R_{Q S}+\sum_{C S} C S R_{D R}\left(t-t_{C}\right)\right)
$$

where $C S R_{D R}$ designates the deficiency (i.e., dynamic) portion of the critical-state response. Both the regular contributions and critical-state contributions are stored at each time step for each valid $\mathrm{DEP}_{\mathrm{i}} / \mathrm{DOF}_{\mathrm{j}}$ combination.

\section{SAMPLE CASES}

In this section, we present the results of four example runs illustrating the capabilities of the IP code. The first example exercises the code in linear indicial theoretical mode. The second example illustrates the effect of interpolation accuracy for the case of a nonlinear quasistatic prediction involving two degrees of freedom. The third example illustrates the effect of partitioning without any critical states. The last example models hysteresis with two critical states.

\section{$\underline{\text { Linear Indicial Response }}$}

A single input, single output, linear model was constructed using a single indicial response node. The indicial response is of simple exponential form $\tilde{f}=\left(1-e^{-t / \tau}\right)$, so that the response $f(t)$ to an input $\alpha=4 e^{i \omega t}$ is known analytically. Figure 5 shows the result of the IP code, run with the periodic option. The results $\left(\mathrm{DEP}_{1}(\mathrm{t})\right)$ are plotted as a function of the damping ratio $\tau / T$, where $T$ is the period of the excitation. According to theory, the amplitude and phase shift of the output are those given in the following table:

\begin{tabular}{|lll|}
\hline Ratio $\tau / \mathbf{T}$ & Amplitude & Phase \\
0 & 4 & $0^{\circ}$ \\
0.125 & 3.146 & $-38.1^{\circ}$ \\
0.25 & 2.149 & $-57.5^{\circ}$ \\
0.5 & 1.214 & $-72.3^{\circ}$ \\
1 & 0.629 & $-81.0^{\circ}$ \\
\hline
\end{tabular}

The results shown in Figure $\mathbf{5}$ are exact to numerical accuracy.

\section{Nonlinear Quasistatic Response}

In this example, there are two degrees of freedom, denoted $\alpha$ and $\phi$. However, we assume that the dependent variable denoted $\mathrm{DEP}_{1}$ is a function of $\alpha$ only. The indicial response of $\mathrm{DEP}_{1}$ with respect to $\alpha$ is assumed to be parameterized by both $\alpha$ and $\phi$, 


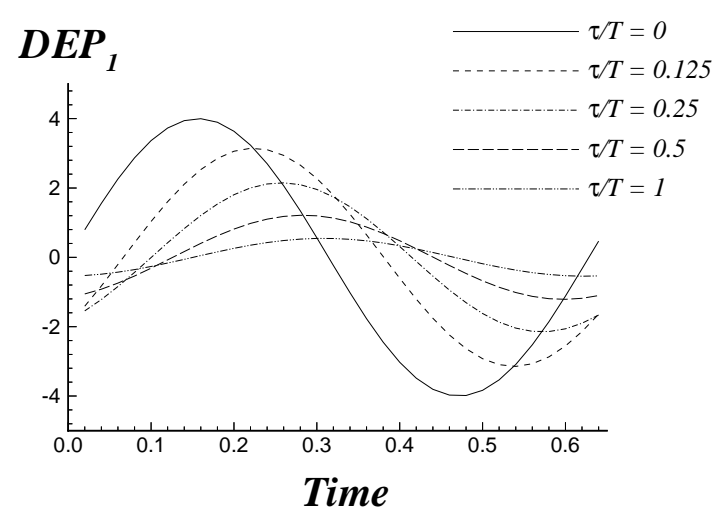

Fig. 5. Linear Indicial Prediction: Effect of Damping Ratio.

however. The maneuver consists simultaneously of a hyperbolic tangent-shaped ramp in $\alpha$ and an impulsive constant roll rate for $\phi$. Its trajectory (in $\alpha-\phi$ parameter space) is shown in Figure 6, along with the location of the six nodal indicial responses used (three at $\phi=0$ and three at $\phi=50$ ). In this exercise, the deficiency responses are all assumed to be zero (quasistatic prediction).

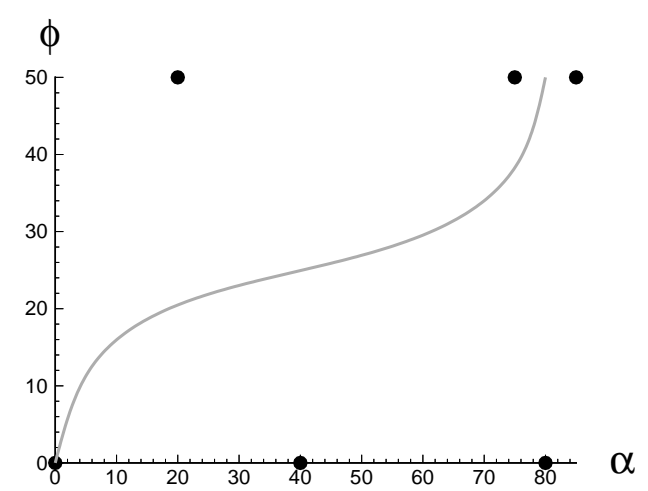

Fig. 6. Example \#2: Nodal Responses and Maneuver Trajectory in $\alpha-\phi$ Parameter Space.

In general, it is difficult to make comparisons against exact solutions in the nonlinear case (i.e., the case where the indicial response varies along the trajectory). In some particular cases, however, it is possible to obtain such solutions analytically. One example considered here is when the value of the indicial response is proportional to $\alpha$. In this case, Eq. (2) amounts simply to an integral of the product $\alpha \dot{\alpha}$. With a zero initial condition, the result must then be proportional to $\alpha^{2}$, regardless of the details of the path. This is indeed verified, and is illustrated by the results of Figure 7. Additionally, Figure 7 compares the relative accuracies of two interpolation schemes. The first one (curve labeled '2d.xg') corresponds to the full two-dimensional interpolation in $\alpha-\phi$ parameter space using Shepard quadratic interpolation. The second scheme ('1d.xg') is the result of projecting all nodes on the $\alpha$ axis (a permissible modeling option, since the indicial responses are, by construction, not a function of $\phi$ ). In the latter case, the one-dimensional interpolation is carried out using a cubic spline. The result of the one-dimensional interpolation is seen to be slightly more accurate than the full two-dimensional parameterization/interpolation. Since both interpolation schemes are capable of representing quadratic behavior exactly, the difference in accuracy is due to the node sparseness factor, i.e., the fact that, in two dimensions, on the order of $6^{2}$ nodes are required to achieve the same node density as in the one-dimensional case. This illustrates the fact that reduced parameterization should be used whenever possible.

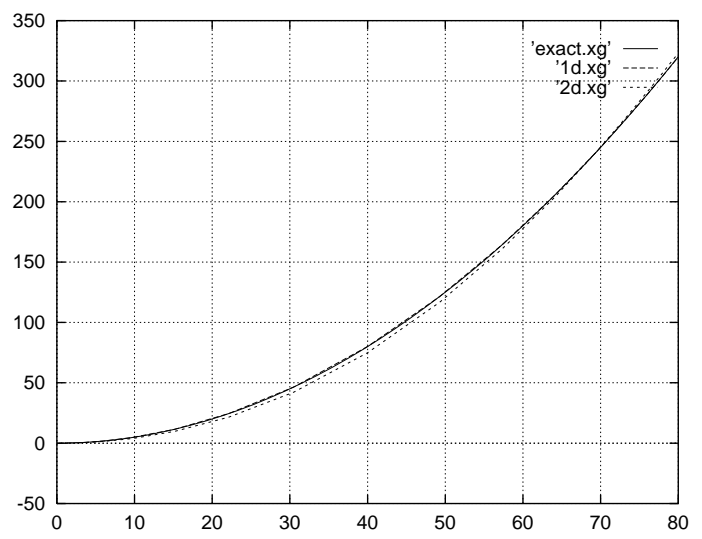

Fig. 7. Quasistatic Quadratic Prediction of $D E P_{1}$ vs. DOF $=\alpha$ : Comparison Between 2-D and 1-D Interpolation Schemes.

\section{IR Space Partitioning}

We now consider a case where the indicial response space is partitioned. The hypothetical system has a single degree of freedom $\mathrm{DOF}_{1}=\alpha$ and a single dependent variable, denoted $\mathrm{DEP}_{1}$. The indicial response of $\mathrm{DEP}_{1}$ with respect to $\mathrm{DOF}_{1}$ is assumed to be parameterized by $\alpha$ and $\operatorname{sgn}(\dot{\alpha})$, and there are no critical states. The location of the available nodal indicial responses is indicated in Figure 8. It is assumed that the IR space has two partitions: one corresponding to $\alpha<35$, and the other corresponding to $\alpha>35$. For simplicity of interpretation, all nodes in a given partition are chosen to be identical to each 
other. For $\alpha<35$, they are given by $\tilde{f}_{1}=\left(1-e^{-t / \tau} 1\right)$, while for $\alpha>35$, they are given by $\tilde{f}_{2}^{1}=2\left(1-e^{-t / \tau}\right)$. In each case, the excitation $\alpha(t)$ is assumed to be sinusoidal: $\alpha(\mathrm{t})=40+40 \sin (\omega \mathrm{t})$.

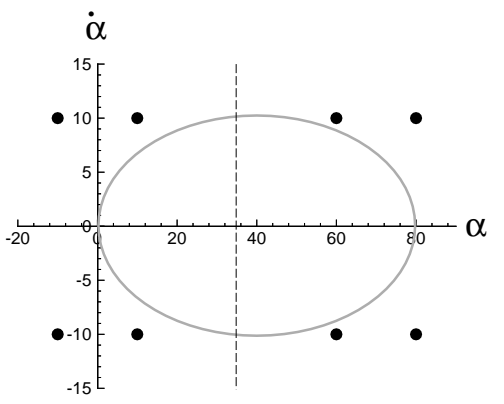

Fig. 8. Example \#3: Nodal Responses and Maneuver Trajectory in $(\alpha, d \alpha / d t)$ Parameter Space. (Dashed vertical line delimits space partitions).

Let us first consider the "fast response" limit $\left(\tau_{1}\right.$, $\tau_{2} \rightarrow 0$, or $\tilde{h}_{1}=\tilde{h}_{2}=0$ ). In this case, one would expect $\operatorname{DEP}_{1}(\alpha)$ to collapse onto a single curve made of two linear segments, characterized by $\mathrm{d}\left(\mathrm{DEP}_{1}\right) / \mathrm{d} \alpha=1$ for $\alpha<35$, and $\mathrm{d}\left(\mathrm{DEP}_{1}\right) / \mathrm{d} \alpha=2$ for $\alpha>35$. This corresponds to the curve labelled 'two_part.xg' in Figure 9. By contrast, note that, if the partition is removed so that all nodes are considered together in the interpolation process, there is a gradual blending of the IRs, as demonstrated by the curve 'single_part.xg.'

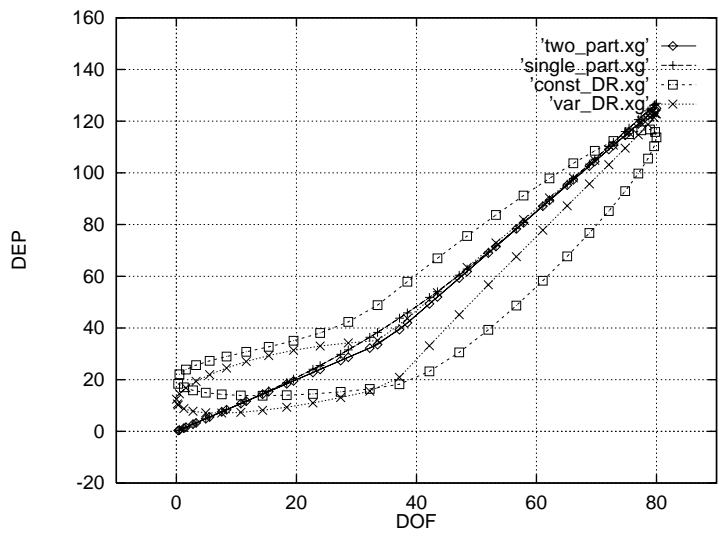

Fig. 9. Nonlinear Indicial Prediction: Effect of Parameter Space Partitioning.

Consider now the case of an unsteady prediction, where the deficiency responses (once normalized by the quasistatic IR value) are identical on both sides of the partition. In other words, $\tau_{1}=\tau_{2}$. The resulting prediction, for $\tau \omega \approx 0.785$, is shown in Figure 9 (curve labelled 'const_DR.xg'). If, on the other hand, $\tau_{2}$ is reduced by a factor of approximately 30 , so that $\tau \omega \approx 0.025$ for the right-hand side partition $(\alpha>35)$, then the result labelled 'var_DR.xg' is obtained. In both cases, the loops are traveled in the counterclockwise direction. Note, in particular, that the 'var_DG.xg' prediction does not immediately join the quasistatic curve, since there are memory effects associated with the trajectory having previously visited the left-hand side partition, where strong dynamic effects were present.

As previously mentioned, it is difficult, in the unsteady nonlinear case, to compare the predictions against analytical solutions. However, it is possible to infer analytical characteristics by reasoning on the invidual lobes of the dynamic hysteresis curves. For example, in the case where $\tau_{1}=\tau_{2}$, the $T / \tau$ ratio exceeds eight. It can be argued, therefore, that most (> 85\%) unsteady effects caused by the previous partition are "forgotten" by the time the trajectory reaches the end lobes, since these are reached approximately two time constants after crossing the partition. For $\tau \omega \approx 0.785$, theory predicts that the amplitude of the output is approximately $80 \%$ of its quasistatic counterpart. Theory also predicts that the angle of the ellipse will tilt by $-23 \%$ in the left-hand side partition, while for the right-hand side partition, the angle tilts down by less than 5\%. The resulting nonlinear 'folding' is indeed observed in the figure. The thickness of the ellipses is predicted to be around 27\%. Actual thicknesses (as inferred from measurements made at the half major axis location) are around $24 \%$.

\section{Critical State Hysteresis}

In this example, we reproduce with the IP code one of the results published in Reference 9. An artificially constructed nonlinear system was designed to mimic the rolling moment coefficient response of the $65^{\circ}$ delta wing undergoing forced roll oscillations. In Reference 9 we considered small amplitude oscillations in the range of $-4^{\circ} \leq \phi \leq 8^{\circ}$, for a support sting angle of $30^{\circ}$ and at a freestream Mach number of three-tenths. Static data taken at fine roll increments [5,10] suggest the existence of critical state transitions at $\phi=5.20^{\circ}$ and $\phi=4.67^{\circ}$ for increasing and decreasing $\phi$, respectively.

The real data were idealized using a nonlinear indicial model (described below), and it is this idealized 
model which we consider here. The model is a single input, single output, model. The indicial responses are parameterized by $\phi$ (the degree of freedom) only, with nodal indicial responses defined at $\phi=-4^{\circ}$, $-1.3^{\circ}, 1.6^{\circ}, 4.6^{\circ}, 5.3^{\circ}$, and $8.6^{\circ}$. In addition, two jump responses are defined at the crossing of critical states, i.e., at $\phi_{\mathrm{CS}}=5.2^{\circ}$ for $\mathrm{d} \phi / \mathrm{dt}>0$, and at $\phi_{\mathrm{CS}}=4.7^{\circ}$ for $\mathrm{d} \phi / \mathrm{dt}<0$. The various time constants were chosen so as to qualitatively reproduce some of the actual hysteresis loops recorded in this roll angle range [9]. The indicial and critical-state responses contained in the database are as follows:

\begin{tabular}{|ccc|}
\hline$\phi$ & Type & \multicolumn{1}{c|}{ Expression } \\
-4 & IR & $2.5-3.5 \exp (-\mathrm{t} / 1.2)$ \\
-1.3 & IR & $-0.5-0.5 \exp (-\mathrm{t} / 0.4)$ \\
1.6 & IR & $-0.5-0.5 \exp (-\mathrm{t} / 0.4)$ \\
4.6 & IR & $1.3529-2.3529 \exp (-\mathrm{t} / 0.4)$ \\
5.3 & IR & $1.6-2.3833 \exp (-\mathrm{t} / 0.4)-0.2167 \exp (-\mathrm{t} / 0.6)$ \\
8.6 & IR & $1.6-2.6 \exp (-\mathrm{t} / 0.6)$ \\
4.7 & CSR & $-2.5+12.5 \exp (-\mathrm{t})-10 \exp (-\mathrm{t} / 0.76)$ \\
5.2 & CSR & $2.5-12.5 \exp (-\mathrm{t})+10 \exp (-\mathrm{t} / 0.76)$ \\
\hline
\end{tabular}

In Reference 9, the result of extracted indicial and critical-state responses was used to predict the hysteresis loops associated with various novel maneuvers. The one considered here is $\phi=2+8 \sin (\omega \mathrm{t})$, with $\omega=2.0$. This corresponds to the prediction labeled ' $\mathrm{k}=0.0267$ ' in Fig. 18 of Reference 9. This previous prediction is shown in

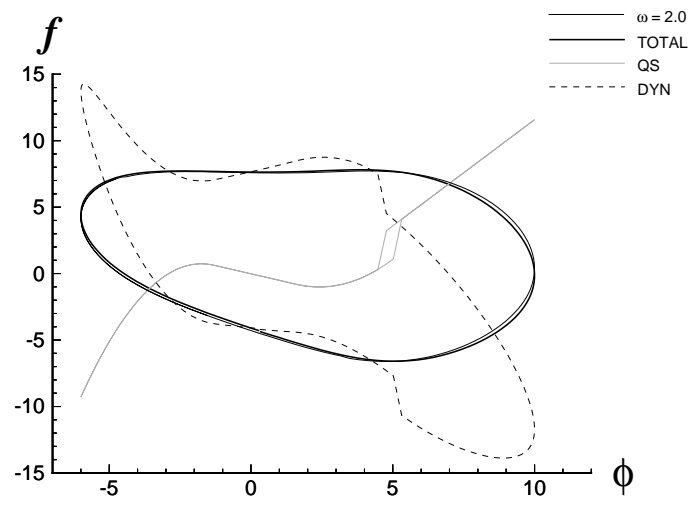

Fig. 10. Indicial Theoretical Prediction Components for a Nonlinear Dynamic Case with Static Hysteresis. (The curve labeled ' $\omega=2.0$ ' corresponds to a previous prediction made in Reference 9 (Fig. 18) using a different code).

Figure 10, where it is compared to the prediction made using the IP code (curve labeled 'TOTAL'). In addition, the output of the code allows the examination of the various prediction components.
For example, the quasistatic and dynamic components of the prediction are indicated in Figure 10. Note that the dynamic component includes both the IR and CSR deficiency response contributions. A more detailed analysis reveals that, at the relatively high frequency of the excitation, the net build-up due to jump responses is relatively small, due to a cancellation effect between consecutive critical states. Both prediction methods assume a piecewise linear interpolation between the indicial responses. There are, however, differences in the quadrature method used for time integration.

\section{ALGORITHM CAPABILITIES}

The core computational engines of the indicial prediction module consist of quadrature and interpolation operations. For a given set of modeling decisions (parameterizations) and a given database density, the quadrature and interpolation operations directly affect the accuracy of the prediction. It is important, therefore, to discuss the various options available. Each engine is implemented in shared object form and, thus, is external to the program, allowing IPS's versatility to continually improve, as shared objects are added on.

At present, the quadrature shared objects include the following: midpoint rule integration, adaptive trapezoidal integration, adaptive Simpson integration, a high-order adaptive integration method, and a Dirac integration method by midpoint rule. The latter allows the IPS to be used when the inputs are discontinuous in time (thus generating Dirac deltas in the integrand of the convolution integrals), such as with square wave or step inputs.

The interpolation shared objects are categorized according to dimensionality and whether the interpolation method is restricted to ordered data on a lattice or functions on scattered nodes. The present interpolation capabilities include, in one dimension, piecewise linear interpolation as well as a variety of univariate splines (natural, FMM, shape-preserving, improved Akima, and Nielson). For bivariate interpolation, five different interpolators are provided: four scattered data interpolators, and one sorted node interpolator. The bivariate scattered data interpolators include: modified Shepard quadratic, SrfPack linear, SrfPack nonlinear, and Akima bivariate cubic. The sorted data algorithm is a bivariate bicubic interpolator. A trivariate scattered data interpolator will be made available for three-dimensional 
interpolation in the near future. In addition, an arbitrary-dimensional scattered data interpolation based on reciprocal multiquadrics has also been implemented.

Together, these capabilities provide the Indicial Prediction System with a wide range of modeling options.

\section{CODE VALIDATION}

The validation of the nonlinear indicial concept has previously been discussed in References 6 and 7 . Further validation examples are provided here for completeness.

The first example concerns the application of the IP program to the case of an artificial neural network trained on wind tunnel data of a pitching wing undergoing dynamic stall [11]. It was previously shown [7] that this nonlinear system includes at least one critical state, which is associated with crossing the static stall angle. The trained neural network is used here as a nonlinear plant, taken to represent accurately the aerodynamic behavior (five sectional force coefficients) associated with a rectangular wing pitching from 0 to 60 degrees.

The nonlinear indicial modeling of this system is characterized by the following: (i) a critical state between 16 and 17 degrees angle of attack (aerodynamic bifurcation associated with static stall), (ii) nine indicial responses in the region prior to encountering the critical state $(\alpha<16 \mathrm{deg}$.), and (iii) twenty-nine indicial responses in the post criticalstate region. The nodal responses are scattered in a two-dimensional space characterized by instantaneous angle of attack and pitch rate. In addition, since the critical-state response changes with pitch rate, it is represented using three nodal responses.

Figure 11 compares the prediction made with the new code (labeled 'IP (1997)') to that made using a slightly different treatment of the critical-state encounter (labeled 'mcvJ (1995),' from Ref. 7). Thus, we do not expect perfect agreement between the two methods. Nevertheless, the scattered two-dimensional interpolation method and quadrature method were matched, and the resulting comparisons are shown in Figure 11. The simulated wing motion in this figure corresponds to a nominally constant pitch rate maneuver. The figure depicts the total sectional lift coefficient build-up (thick solid line, labeled
'TOTAL'), as well as the individual contributions due to the regular (convolution integral) contributions ('IRTOT') and the jump (critical-state) response contributions, labeled 'CSRTOT'.

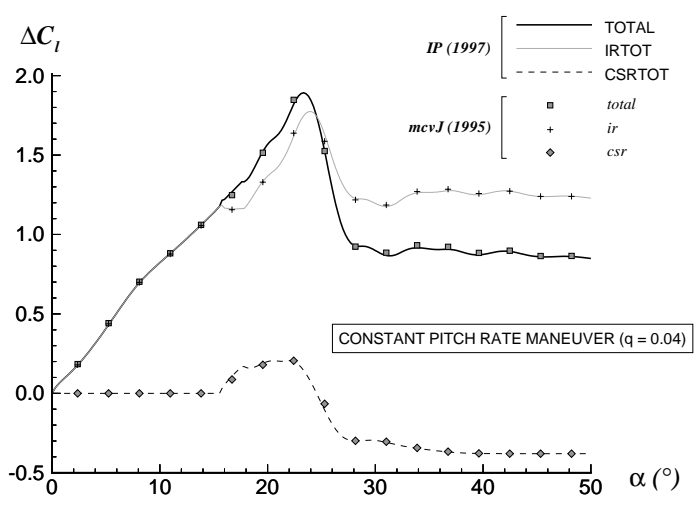

Fig. 11. Comparison of Indicial Predictions Between 'New' (IP) Code and 'Old' (mcvJ) Code for the Neural Network Simulation of a $60^{\circ}$ Pitch Up Maneuver (Rectangular Wing) at Constant Rate.

The second validation example is the application of the method to the prediction of the rolling moment, $\mathrm{C}_{1}$, of a 65-degree sweep delta wing. For the dynamic cases discussed here, the delta wing body axis is held at a 30 degree angle to the freestream, and the Mach number is approximately three-tenths. The wing undergoes forced rolling motions $\phi(t)$, and the measured aerodynamic force coefficient time histories are recorded. These data are part of a comprehensive database collected under a joint program involving the U.S. Air Force Research Laboratory (formerly USAF/WL) and the Canadian Institute for Aerospace Research (IAR).

The physics of the flowfield and the aerodynamic forces generated by the forced roll oscillations on the 65-degree delta wing have been the topic of numerous papers over the years $[4,5,10,12-14]$. In particular, the critical states of the rolling moment curve are well-documented, and have been identified through discontinuities of the static $\mathrm{C}_{1}$ vs $\phi$ curve, Figure 12. Myatt [12] used parameter identification techniques to determine the indicial and critical-state responses of the rolling moment with respect to roll angle. The work of Reference 12 provides analytical approximations for the critical-state responses at $\phi=-11^{\circ},-8.25^{\circ},-4^{\circ}, 5^{\circ}, 8.5^{\circ}$ and $11.3^{\circ}$. In between critical states, the indicial responses assume the following form: 


$$
\begin{aligned}
\frac{\delta C_{1}}{d \phi} & =\frac{d C_{1_{Q S}}}{d \phi}+\left\lfloor C_{1_{p}}^{p}+\frac{\alpha_{1}}{\beta}\right\rfloor \delta(t) \\
& +\left[\frac{\alpha_{0}-\alpha_{1} / \beta}{\beta}-\frac{d C_{1_{Q S}}^{V}}{d \boldsymbol{\phi}}\right] \exp (-t / \boldsymbol{\beta})
\end{aligned}
$$

where $d c_{1} / d \phi$ represents the slope of the quasistatic rốlling moment curve with respect to $\phi$, and $d C_{1}^{v} / d \phi$ is the vortical component of $d C_{1} / d \phi^{Q S}$. The quasistatic curve $C_{1}$ is known (fitted) from experiment, and $C_{1}^{V}$ is inferred from $C_{I}$ after calculating the potentiall flow component using QUADPAN [15]. The nonlinearity in Myatt's model comes from the variation in the static slopes and from the existence of critical states. In between two critical states, the parameters $\alpha_{0}, \alpha_{1}$ and $\beta$ remain constant.

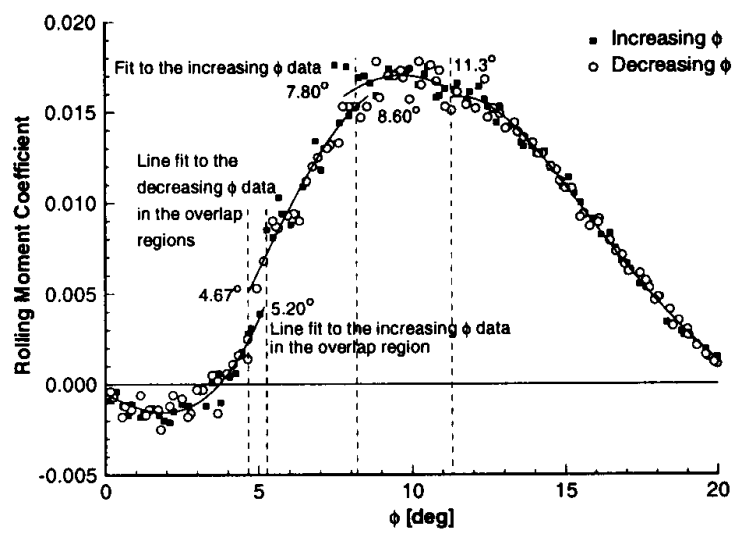

Fig. 12. Time-Averaged Static Rolling Moment Coefficient for both Increasing and Decreasing $\phi$ (from Ref. 10).

Myatt's NIR model thus provides a unique opportunity to validate the IP code on a problem of interest, by using different methods to carry out the prediction calculations. The modeling in Myatt's method and in the IP code is identical. By this we mean that, for validation purposes, the IR and CSR nodes used in the IP code are those of Myatt. Furthermore, the parameter space is partitioned in the same way as in Myatt's representation (i.e., a total of twelve critical-state responses: six for positive roll rate, and six for negative roll rate).

The primary difference between the two models is that, whereas the Myatt model has a complete and continuous "knowledge" of the indicial responses everywhere, the IP program is based on parametric interpolation of approximately 30 (nodal) indicial responses, which are known only at discrete values of the parameter space). Other differences include the fact that, in IP, the "stationary" limit cycle behavior (in the case of periodic maneuvers) is computed directly, whereas Myatt's method integrates forward, starting from some assumed equilibrium point. Finally, Myatt's implementation is based on an equivalent state-space form of the aerodynamic model, resulting in a computationally efficient differential (rather than integral) form for the equations.

Some typical validation results are given in Figures 13 through 16, indicating good agreement between the two methods. In the figures, the label 'analytical model' refers to Myatt's prediction.

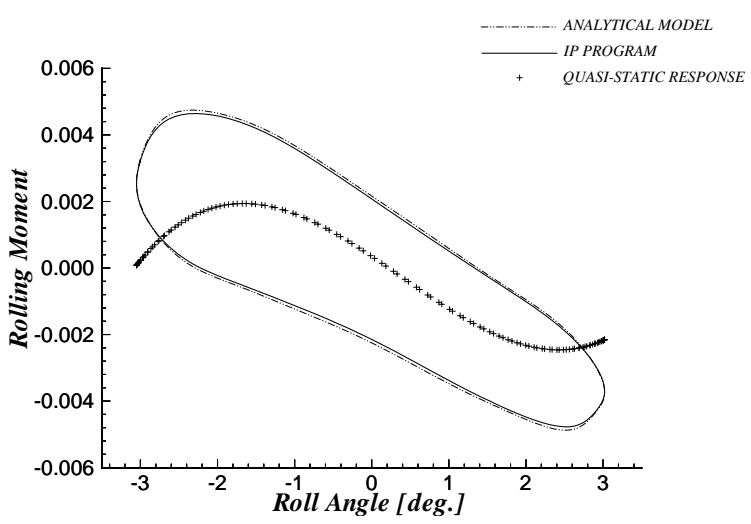

Fig. 13. Predicted Rolling Moment Response for $\phi=0 \pm 3$ deg., $k=0.02$.

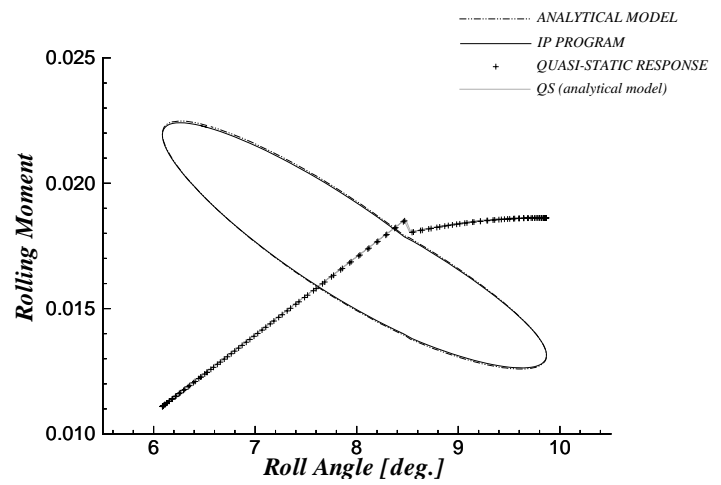

Fig. 14. Predicted Rolling Moment Response for $\phi=8 \pm 2$ deg., $k=0.14$. 


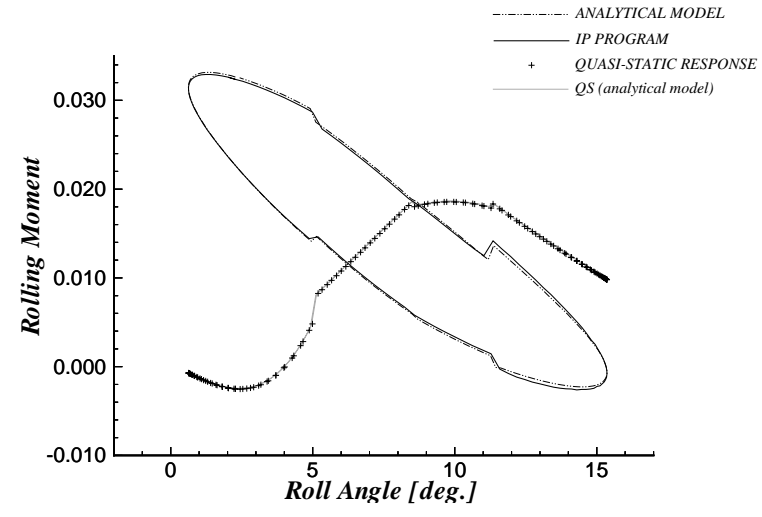

Fig. 15. Predicted Rolling Moment Response for $\phi=8 \pm 8$ deg., $k=0.14$.

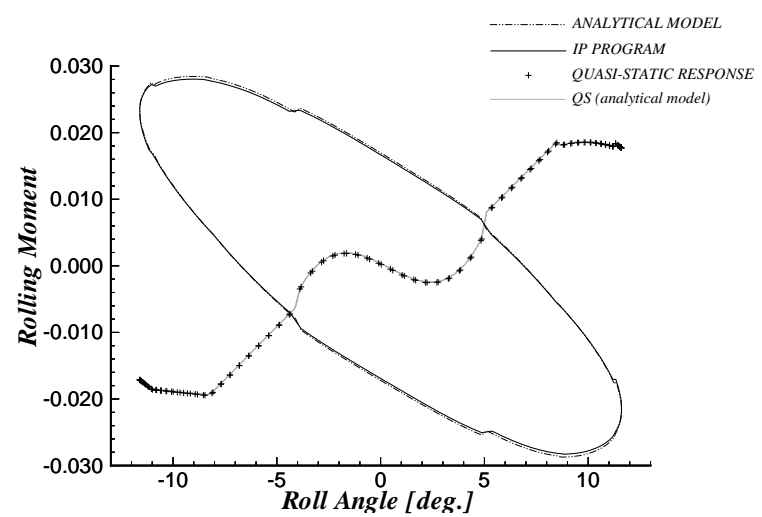

Fig. 16 Predicted Rolling Moment Response for $\phi=0 \pm 12$ deg., $k=0.08$.

\section{RESPONSE KERNEL EXTRACTION / RESULTS}

An important capability of the Indicial Prediction System is its ability to extract nonlinear indicial and critical-state responses from empirical data. Such a capability is necessary because it is, in general, difficult to obtain the indicial responses of a system directly. The description of the nonlinear indicial and critical state extraction scheme will be the topic of a separate paper [8]. Sample extraction results are presented here, since it is only together that the prediction (IP) and extraction (IE) modules make the IPS a true data-based prediction method. From a functional point of view, the method is similar to a neural network: sample maneuvers (input/ouput transfer functions) can be supplied as "training" data.
These training data are used to extract the response kernel of indicial and critical-state responses. This database kernel, in turn, is used to predict the system's response to arbitrary inputs.

To illustrate how the system works, we will, again, consider the forced rolling motions of the 65-degree delta wing. This time, however, the indicial responses are extracted from the data, rather than using Myatt's analytical representation. (The present results pertain to a small range of roll angles $\left(-4.05^{\circ} \leq \phi \leq 5^{\circ}\right)$ without any critical-state transitions).

First, two essential properties of the extraction method are illustrated. The first one is robustness with respect to noise. The second is the convergence property.

Figure 17 depicts the results of a series of error metrics tests in which the amount of noise in the training data is varied in a controlled manner, through the number $n_{\text {harm }}$ of retained harmonics. The extracted nodes are then used to predict the very maneuvers they were "trained" on. The resulting total norm-2 (rms) error is shown in Figure $\mathbf{1 7}$ as a function of $n_{\text {harm }}$. For reference, the error associated with the prediction made using Myatt's IR nodes is indicated by the dashed line and circles. The insets represent typical data for various levels of filtering. The error associated with the extracted nodes is indicated by the

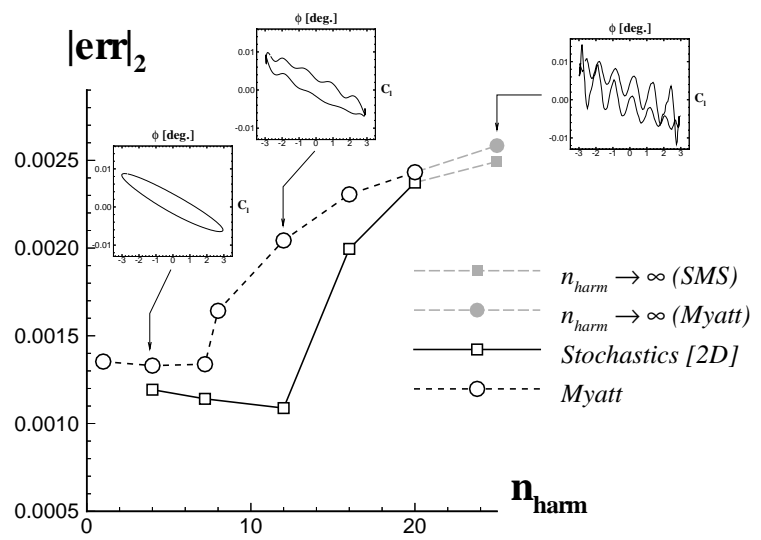

Fig. 17. Effect of Noise on Accuracy of Extraction. (Insets depict a typical data set for a given noise level, characterized by the number of retained harmonics). 
solid line and square symbols. In each case, the filled-in grey symbols represent the error when using the raw data (denoted $n_{\text {harm }} \rightarrow \infty$ ).

The error corresponding to the extraction (square symbols) is both lower than the error associated with Myatt's prediction, and remains flat or slightly decreasing until $n_{\text {harm }}>12$. Only after at least 12 harmonics are retained in the data does the error increase. The flat portion of the curve illustrates the fact that the IR nodes can successfully be extracted for varying levels of noise. Thus, the method appears to be robust with respect to noise.

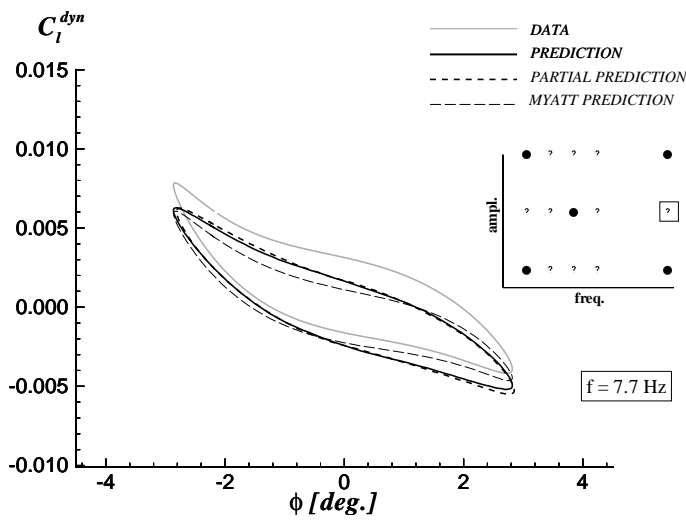

Fig. 18. Nonlinear Indicial Prediction of $f=7.7 \mathrm{~Hz}$, $\phi= \pm 3^{\circ}$ Data Based on Extraction Using (a) Full Data Set (Solid Line), and (b) One Third of the Data (Short Dashes). (Inset indicates the predicted motion parameters with respect to those of the partial training data set; Myatt prediction indicated for reference).

The convergence property is defined as follows. To demonstrate that the IR extraction is not merely data fitting but does indeed have predictive value, we must be able to extract the nodal responses with sufficient accuracy from a partial data set. Furthermore, the indicial responses must approach those obtained using the full data set as the decimation is reduced. The basic idea is to attempt to extract indicial responses using only a portion of the data available and to subsequently verify the predictive potential of the method on maneuvers which were not part of the training data set. This methodology is similar to that used with artificial neural networks and other databased prediction methods.
The convergence property will be elaborated upon in Reference 8. The sample results of Figures 18 and 19 are provided as illustrations. Figure 18 compares full- and partial-data set predictions of the dynamic rolling moment response for a harmonic motion not included in the training. Three predictions are shown: the first (solid black line) is the IP prediction based on the nodes extracted using all 15 harmonic maneuvers available; the second (dashed line) is the prediction when only five maneuvers are included in the training data set; the third prediction is the Myatt prediction, which is included for reference. The apparent shift between the data and the various nonlinear indicial predictions has been observed previously, and the exact cause of this discrepancy is not known. (Some of the possibilities include optically encoded roll angle measurement error and/or the presence of nonlinear rate effects). The important point of Figure 18 is that the partial prediction, which is based on five maneuvers, is almost identical to the prediction based on extraction using the full data set.

Since the use of harmonic motion data for the extraction is known to represent a "worst case scenario" [8], a good test of the method is, first, to extract the nodes using harmonic data, and then to attempt to predict the ramp-and-hold data using the extracted nodes. Figure 19 shows the nonlinear indicial prediction of the rolling moment for a ramp motion from $\phi=-4^{\circ}$ to $\phi=+4^{\circ}$, with a maximum roll rate of approximately $60 \mathrm{deg} . / \mathrm{s}$. The result of the extraction method (long dashes, labeled 'SMS') is compared in this plot to the reference prediction of Myatt (short dashes). The SMS extraction-based prediction uses cubic spline interpolation. A

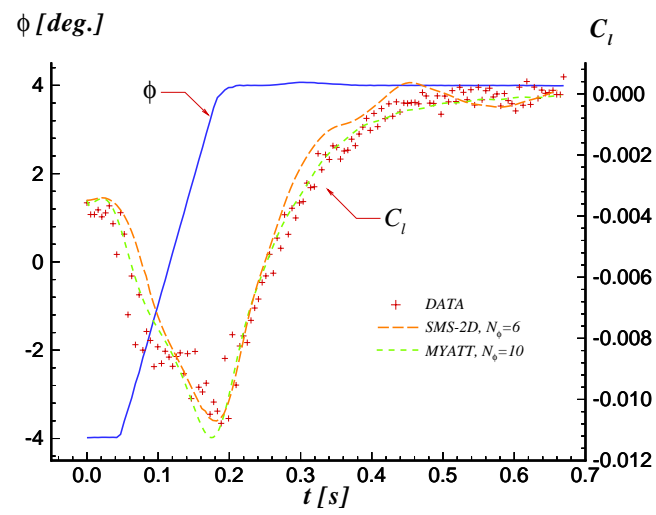

Fig. 19. Prediction of $\phi=-4^{\circ}$ to $+4^{\circ}, 60^{\circ} / \mathrm{s}$ Ramp Maneuver. The IE/IP (label: 'SMS') Prediction is Based on Harmonic Data Only. 
comparison with the same prediction using linear interpolation (not shown) indicates that the IE/IP prediction is accurate, provided that one uses the higher-order interpolation scheme on the static component. This is so, because of the relatively small number of IR nodes in this region [8]. With cubic interpolation, the IE/IP prediction is seen to be similar to Myatt's. This result is significant, because the nodes were extracted on the basis of harmonic data only.

While predictions using only a partial data set are typically less accurate, the results of Figures 18 and $\mathbf{1 9}$ confirm the predictive ability of the method.

\section{CONCLUDING REMARKS / SUMMARY}

Nonlinear indicial response theory addresses the need for high-fidelity prediction of nonlinear unsteady aerodynamic characteristics. The present paper provides an overview of the Indicial Prediction System as a tool kit for the aerodynamicist. Synthetically constructed examples are used to illustrate the modeling capabilities of the system, and code validation examples are provided. The complete system (nonlinear response kernel extraction from experimental data, followed by indicial prediction for novel maneuvers) is demonstrated for the rolling moment of a 65-degree sweep delta wing in rolling motion.

\section{ACKNOWLEDGMENT}

The support of this work by the Air Force Research Laboratory Flight Control Division under Phase II SBIR Contract F33615-96-C-3613 is gratefully acknowledged.

\section{REFERENCES}

[1] Tobak, M., Chapman, G. T., and Schiff, L. B.: Mathematical Modeling of the Aerodynamic Characteristics in Flight Dynamics, NASA TM 85880, 1984.

[2] Tobak, M. and Chapman, G. T.: Nonlinear Problems in Flight Dynamics Involving Aerodynamic Bifurcations, NASA TM 86706, 1985.

[3] Nixon, D.: “Alternative Methods for Modeling Unsteady Transonic Flows," Unsteady Transonic Aerodynamics, Vol. 120 of Progress in Astronautics and Aeronautics, Ed. by D. Nixon, AIAA, 1989.
[4] Jenkins, J. E., Myatt, J. H., and Hanff, E. S.: "Body-Axis Rolling Motion Critical States of a 65-Degree Delta Wing," J. Aircraft, Vol. 33, No. 2, 1996, pp. 268-278.

[5] Jobe, C. E., Hsia, A. H., Jenkins, J. E., and Addington, G. A.: "Critical States and Flow Structure on a 65-Deg Delta Wing," J. Aircraft, Vol. 33, No. 2, 1996, pp. 347-352.

[6] Reisenthel, P. H.: Development of a Nonlinear Indicial Model For Maneuvering Fighter Aircraft, AIAA Paper No. 96-0896, January 1996.

[7] Reisenthel, P. H.: Application of Nonlinear Indicial Modeling to the Prediction of a Dynamically Stalling Wing, AIAA Paper No. 96-2493, June 1996.

[8] Reisenthel, P. H. and Bettencourt, M. T.: Extraction of Nonlinear Indicial And Critical State Responses From Experimental Data, AIAA Paper No. -, submitted for presentation at the $37^{\text {th }}$ AIAA Aerospace Sciences Meeting and Exhibit, Reno, NV, January 11-14, 1999.

[9] Reisenthel, P. H.: Development of a Nonlinear Indicial Model Using Response Functions Generated by a Neural Network, AIAA Paper No. 97-0337, January 1997.

[10] Grismer, D. S. and Jenkins, J. E.: Critical-State Transients for a Rolling $65^{\circ}$ Delta Wing, AIAA Paper No. 96-2432, June 1996.

[11] Faller, W. E., Schreck, S. J., and Luttges, M. W.: Real-Time Prediction and Control of Three-Dimensional Unsteady Separated Flow Fields Using Neural Networks, AIAA Paper No. 94-0532, January 1994.

[12] Myatt, J. H.: Modeling the Rolling Moment on the 65-Degree Delta Wing for Rolling Motions at High Angle of Attack, Ph.D. dissertation, Department of Aeronautics and Astronautics, Stanford University, April 1997.

[13] Jenkins, J. E. and Myatt, J. H.: Modeling Nonlinear Aerodynamic Loads for Aircraft Stability and Control Analysis, AGARD Report 789, pp. 13/1-13/10, February 1993.

[14] Myatt, J. H.:, Modeling the Rolling 65-Degree Delta Wing with Critical State Encounters, AIAA Paper No. 97-3646, August 1997.

[15] Youngreen, H. H., Bouchard, E. E., and Coopersmith, R. M.: Quadrilateral Element Panel Method: User's Manual, Ver. 3.2, Lockheed LR 30563, Burbank, CA, 1984. 